\title{
Dynamic Gamma Spectral Measurements of Primary Coolant and Piping at Various Operating Nuclear Power Plants
}

\author{
Frazier L. Bronson \\ Mirion Technologies (Canberra) \\ 800 Research Parkway Meriden CT USA
}

\begin{abstract}
An on-going EPRI-supported project involves the use of a collimated CZT gamma spectroscopy detector for quantitative measurements of the gamma spectra from primary coolant piping at various times and locations at several operating NPPs. An automatic system - the Data Analyst takes the spectra from the CZT, and analyzes it on a continuing repeating basis. Measurements are taken simultaneously covering different elapsed times; e.g. 10 minutes for viewing short dynamic events, and 10 hours for low detection limits. The detector is calibrated using the ISOCS mathematical efficiency calibration software, which allows the activity to be computed for a radioactive fluid inside the pipe, or radioactive surface contamination inside or outside the pipe. Measurements have been taken during the reactor shutdown cycle, during the startup cycle, and during continuous full power operations. Examples from these deployments and potential future uses for this technology are described.
\end{abstract}

\section{Introduction}

Most nuclear power plants [NPP] operating today have equipment and operating procedures that were developed many years ago (ref 1, 2, 3, 4). Most radioactivity measurements are either obtained by continuous devices that are not nuclide-specific [dose-rate, effluent count-rate monitors] or by sending staff to obtain the reading or extract a sample for further laboratory analysis.

Here we utilized a device to continuously and sequentially acquire and analyze gamma spectral results in real time. The device is portable and easy to deploy so it can used in most any location of the plant.

\section{Advantages of gamma spectroscopy as compared to dose-rate or count-rate devices}

The primary advantage is that the specific nuclide or a mixture of nuclides causing the count-rate or dose-rate is identified. Knowledge of the nuclide-specific behavior can allow the operator to make better decisions. For instance, knowing the nuclide allows better dose-reduction actions to be taken - e.g. calculation of the proper amount of shielding, or knowing what optimum chemistry conditions should be or if simply delaying the execution of a job is the best strategy (ref 5, 6).

Sometimes knowing the activity a specific nuclide is the important item, not the dose-rate. Knowing when the activity of long-lived hard-gamma constituents [Co-58, Co-60] in the presence of short-lived nuclides in primary coolant at the early phases of shutdown allows subsequent operations to be conducted safely at the appropriate time. Knowing that it is only short-lived nuclides with low dose consequences that are in the gas or liquid effluent stream allows proper offsite dose calculations to be made.

In other situations, knowing the activity of minor constituents of the activity, and the change in that activity over time can be very important. Early detection of traces of certain key nuclides could lead to early detection of fuel cladding damage, or control rod leakage, or moisture carry-over.

Dose-rate measurements vs. time, or total count-rate measurements with time cannot provide this information. Nuclide-specific information can therefore lead to be better decision making. It 
can also provide time and cost savings; no need to take a grab sample for laboratory analysis just because of a high dose-rate or count-rate reading.

\section{Advantages of Continuous Quantitative In-situ Gamma Spectroscopy}

The most common practice to obtain nuclide specific results is to send staff out to the site, physically extract a representative sample, and the transport it back to the laboratory for sample preparation and subsequent analysis. Only at that point can the results can be communicated and the appropriate action taken.

An appropriate in-situ gamma spectroscopy instrument has many benefits:

- Typically assays a larger portion of the material, which makes the measurement more representative.

- Eliminates or minimizes the time and effort for sampling, transport to the lab, sample preparation, and analysis.

- Eliminates or minimizes the potential of accidents or spills in collecting and transporting the sample and in the laboratory analysis.

- Reduces dose to staff from sample collection, transport, and analysis.

- Almost instantaneous availability of the results - as soon as the count has been executed; rather than hours later.

- More frequent data and therefore the ability to detect short-term events that might be missed in the periods between grab samples.

- Allows short-lived isotopes to be measured; if not wanted in some situations, a delay line could be added.

- Allows very low MDAs, as the short measurement times can be integrated for the entire time between successive grab samples.

\section{Acquisition and Analysis System}

The 3 primary types of detectors in common use for gamma spectroscopy are Scintillation, HPGe [High Purity Germanium], and CZT [Cadmium Zinc Telluride].

Figure 1 shows the CZT detector that was chosen for three of the presented applications (ref. 7). Figure 2 shows representative spectra of each of the 3 detector types as all may be utilized in the plant setting depending on the

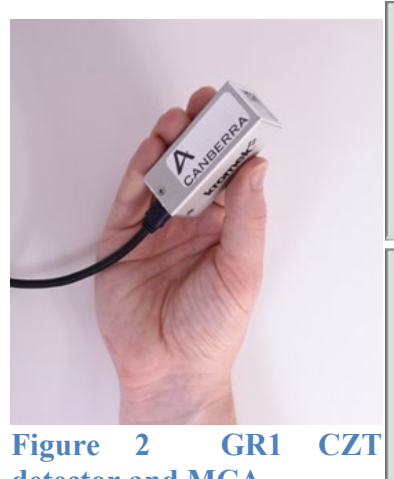
detector and MCA
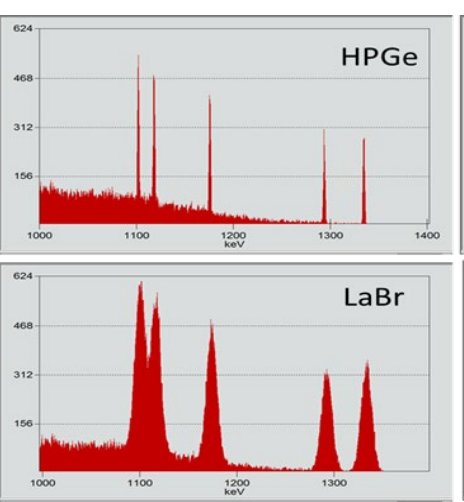

Figure 1 Representative spectra from 4 different detector types. The energy range for all spectra is $1000-1400 \mathrm{keV}$. The nuclides shown are $\mathrm{Zn}-65, \mathrm{Fe}-59$, and $\mathrm{Co}-60$.

The CZT detector in Figure 1, is the Canberra GR1, and with a sensor volume of $1 \mathrm{~cm}^{3}$ and with an integral MCA. The package is only $6.3 \mathrm{~cm}$ long. The device is powered by a USB connection. The primary advantages of this CZT detector are enhanced energy resolution over scintillation detectors, better nuclide identification, and small size for light and low cost shielding.

For very complex spectra it might be better to use HPGe detectors. The major advantages of HPGe detectors are energy resolution, which makes nuclide identification much easier for the software [and human eyes] to interpret, and wide dynamic range of radioactivity that can be 
measured. However the easy deployment of the CZT detector and shield system, coupled with the fact that all spectra would be reviewed by subject matter experts drove the detector choice for these initial EPRI-Mirion-Plant joint projects.

\section{Efficiency Calibrations for Each Geometry}

Although source-based calibrations and MonteCarlo calibrations can be used, the availability of the ISOCS [In-Situ Object Calibration Software] mathematical efficiency calibration software (refs. 9 and 10) makes the required efficiency calibrations much easier for the field practitioner. A detailed detector model is created at the factory and used with MCNP to compute the detector response function for all energies from $15 \mathrm{keV}$ to $7000 \mathrm{keV}$, and for all spatial locations surrounding the detector from zero distance out to 500 meters. The file with these parameters is supplied to the user. The user then adds the relevant sample information and the relationship between the sample and the detector. Twenty-one different

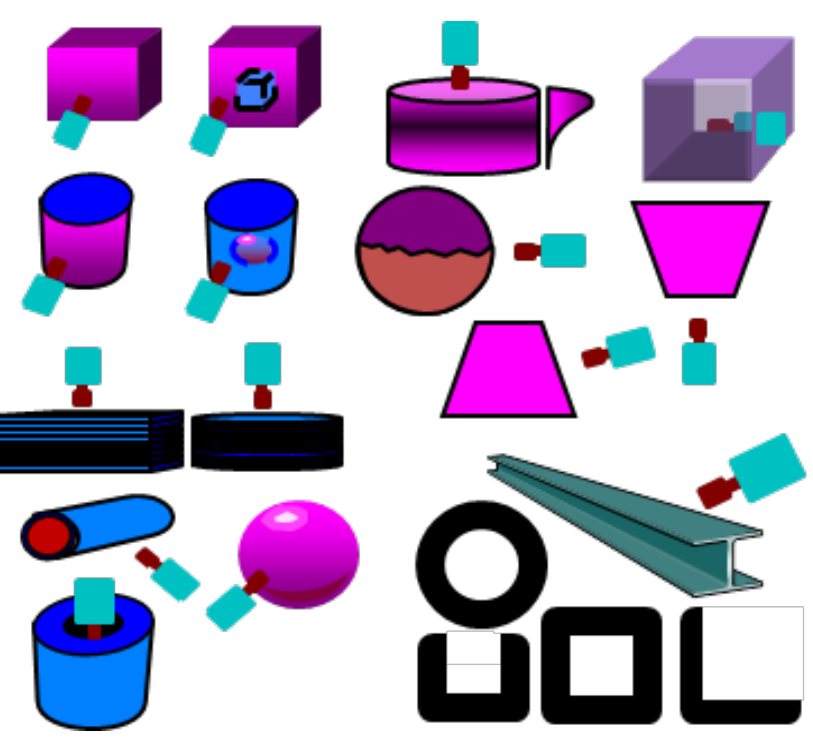

Figure 3 Examples of the various templates available in templates are available covering a wide variety of sample shapes, as illustrated in Figure 3. Each of the templates has a wide amount of flexibility to define the measurement condition. As a very important example in plants, consider pipe measurements. Radioactivity can be deposited on the inside surface, or as a volume source completely or partially filling the pipe, on the outside surface, or as a settled-out deposit (hot spot) on the bottom of the inside of the pipe. All of these examples can be modeled with the software.

\section{Hardware and Software to Control the} Acquisition in Auto-cycling Mode, to Analyze the Spectra, and to Communicate with the Outside World

A device called the Data Analyst [DA] is shown in Figure 4 (ref. 11). It is the small box at the lower right. Only the 3-part combination of a detector, MCA, and the DA is needed to collect and analyze a continual sequence of gamma spectra.

Also shown in Figure 4 is a Tungsten shield and collimator designed to house the CZT detector/MCA when needed. It was used in all of

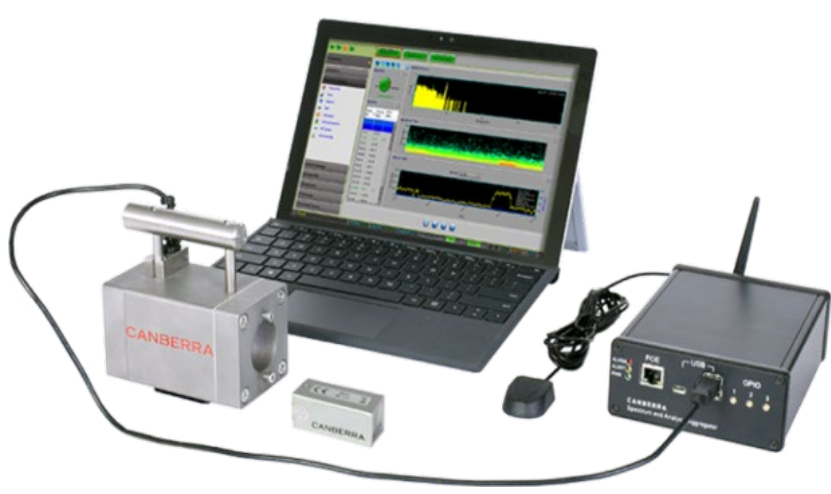

Figure 4 Data Analyst [lower right], along with the GR1 CZT detector [lower center], CZT shield and collimator [lower left], and small laptop these NPP deployments.

When power is applied [via POE on the Ethernet port] the system starts up and immediately starts collecting and analyzing spectra. The PC is only required to set up the system, and to view the results.

The DA works also with HPGe detectors and Scintillation detectors, when coupled to the appropriate MCA. In addition, the EcoGamma wide-range dose-rate probe can be attached to record the dose-rate existing during each spectral analysis.

The DA has an integral GPS receiver which can be used to tag each result with the location of the GPS antenna, shown next to the DA in Figure 4. 
The DA can communicate via Wi-Fi, Ethernet, or USB.

The spectra are analyzed with standard full-function Genie-2000 software algorithms. Therefore they can be viewed and re-analyzed by the user with their same software used in the laboratory.

A key feature of the DA is the ability to run multiple parallel workflows. A workflow is a complete sequence of procedures defining acquisition time, libraries, spectral analysis parameters, alarm levels, output formats, and many other items. Multiple workflows allows the ability to operate one with short count times to capture transient events, and simultaneously another with long count times for better statistics and lower detection limits and fewer false positives. Count times can be as short as a few seconds or as long as many days. For example, in one of the primary coolant monitoring campaigns we used 3 parallel workflows of 20 minutes, 4 hours, and 12 hours.

All data is stored internally until deleted. Standard capacity allows over a year of 10 minute HPGe spectra to be stored.

\section{Application Software to View Data as it is Being Acquired, and to View Historical Data}

A key feature for the real-time monitoring is visualization, which is a standard element of the DA's application software that allows it to view data as it is being acquired. While the PC isn't required for the DA to continuously acquire and analyze spectra, it is obviously required to view the results. The PC could be local nearby the detector and the DA, or as remote as the Wi-Fi, Ethernet, or USB connections allow. Figure 5 shows the main screen.

The numbered regions are briefly described below.

1. Workflows; in this examples 3 workflows are simultaneously operating, and the one highlighted in red is the active display.

2. Alarm, Alert, and Fault status indicators; one indicator is in Alert state as shown by the yellow color.

3. Spectrum Window; this is either the currently acquiring spectrum, or a selected spectrum from a

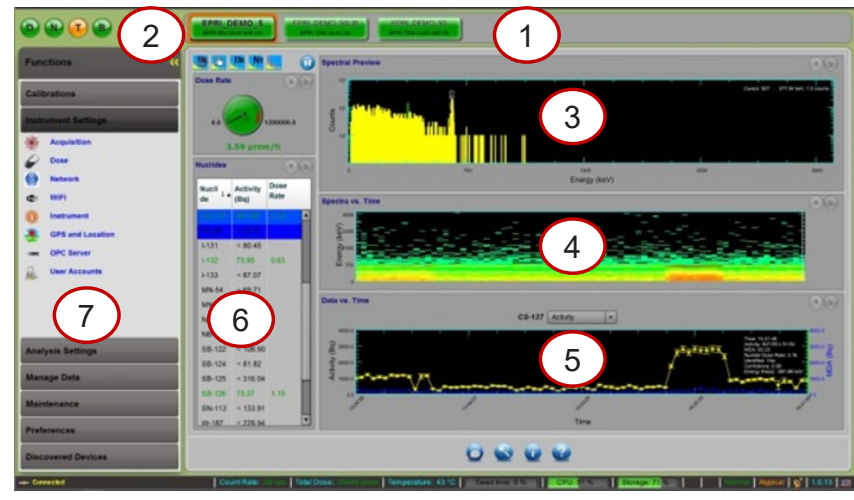

Figure 5 DA acquisition display interface. Numbers are to identify regions discussed in text. previous time.

4. Waterfall Spectra Display Window; this shows a time sequence of the previous spectra that have been acquired in a format that is easy to visually detect changes as shown by the red area toward the right side.

5. Data vs. Time Window; in normal mode this shows a histogram of the counts per second from each acquisition. If the user clicks on a single time period, display window 3 shows the full accumulated spectra for that particular time, and display window 6 shows the nuclide activity results from that particular analysis. If a specific nuclide is selected in Window 6 , then the Data vs. Time histogram changes from cps to Activity for that particular nuclide.

6. Nuclide Activity Window; in normal mode this displays the spectral analysis results of the last assay. Alternately it displays the results of the selected assay from a previous time.

7. Function Window; this is the pathway for the user to access all of the setup, display, archive, calibrate, analyze, and other functions.

Tools to View Current or Past Data, and to Create a Data Base of Results. 
Three such tools are available to the user. One tool is the Data Review section of the Data Analyst. Another tool is a batch review software called Data Prospector. Another tool is the Horizon Supervisory Software.

1. Data Review is part of the DA, and allows the user to select start and stop dates and view the time sequence of key results within that time period.

2. Data Prospector is a separate program running on a local PC that reads the batch/archive output file from the DA. The user then has many options to select from to review the past data and generate output files. The user can also select groups of files and output those nuclide-activity results in a CSV format suitable for import into other programs such as Excel.

3. Horizon Supervisor and Database can be connected full time to the DA, showing the data live as it is acquired. It is designed to be the primary viewing portal for multiple-detector installations. The data is then stored in a database. Key results as specified by the user are easily accessible via SQL commands by other programs.

\section{Field Deployments of Continuous Gamma Spectroscopic Measurements}

The following briefly shows some examples where a spectroscopic detector in a continuous acquisition mode was used at a reactor facility. Figure 6 illustrates the results from the first reactor field deployment, a joint project cofunded by EPRI, Mirion-Canberra, and the Plant a PWR. The graph shows Co-58 concentration in $\mathrm{uCi} / \mathrm{cc}$. The horizontal axis is date. The reactor was shut down at the beginning of day 23. The first forced oxidation was at the beginning of day 24 which caused the spike in Co-58 activity. The blue data are the direct output where the calibration assumes that the entire radioactivity is in the fluid. The red data are the plant grab sample results. Notice that at the beginning the two $r$ The plant coolant activity values are quite low but the in-situ results are not; this is caused by fixed activity incorporation on the pipe's internal surfaces, which is captured in the in-situ readings, but not by the extracted liquid sample analysis. The green data were computed by subtracting a fixed activity value from each of the blue data points. The value was iteratively determined to get results best matching the shape of the plant sample results. This value can then be converted into $\mathrm{uCi} / \mathrm{cm}^{2}$ of internal pipe surface by using the ratio of the two efficiency calibration factors. The Co-58 deposited activity on the pipe's internal surfaces was calculated to be $0.18 \mathrm{uCi} / \mathrm{cm}^{2}$ at the measurement location. This activity can be considered a constant term in this particular case because the piping is in a vertical configuration and the measurement duration is only over a short period in which the isotope is known to be highly soluble, where the probability of additional deposition is minimal.

The green data points were at 5 minute intervals. This allowed observation of the fine

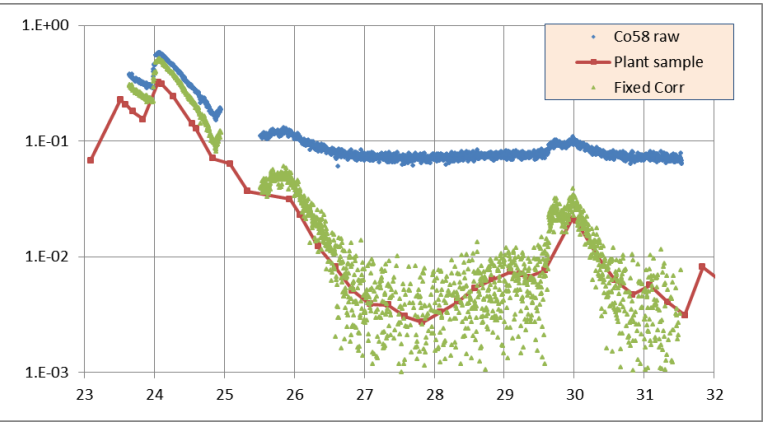

Figure $6 \quad$ Co-58 at first NPP deployment. Blue data are direct measurement results of the pipe. Red data are plant sample results. Green data are activity in the primary coolant fluid derived from the corrected total measurement.

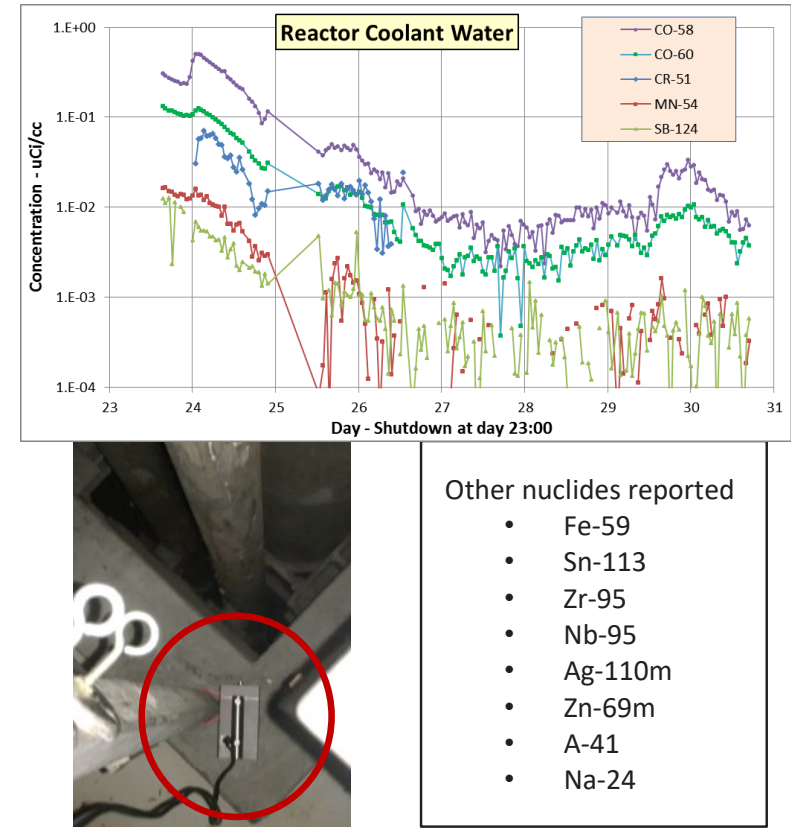

Figure 7 First field deployment at a NPP. Results for each day in top graph. Detector and sample pipe at lower left. Other nuclides seen are shown in table. 
structure in the Co-58 activity, for example the fluctuations around days 26 and 30.

Figure 7 shows an image of the detector - letdown pipe as set up for this test. The nuclide activity graph at the top shows the coolant concentration for 5 key nuclides, after correcting for their respective estimated fixed internal activity. The graph illustrates the disparate behavior between the various nuclides in their response to the forced oxidation. Co-58 has a much higher activity increase on day 24 than does Co-60 and Mn-54. Sb-124 doesn't have any noticeable increase. Eight other nuclides were also observed during these tests.

Figure 8 shows results from the second reactor deployment on the EPRI project, also at a PWR. The graph shows data during the $\sim 30$ day shutdown period, plus a few days before and after. The CZT detector is also aimed at the letdown line. This is the pipe that takes primary coolant to the sample extraction area. The graph shows Co-58 activity in green. The red data is from $\mathrm{F}-18$, a short lived gas that is shown to illustrate reactor power level. The shutdown is on the left, and the stepped startup can be seen on the right. In this case, the primary coolant did not flow continuously through the letdown line during the shutdown. But other outage activities did influence the measurements as annotated in the figure.

Figure 9 shows the results from a third reactor deployment, also from the EPRI project at a PWR. Five key nuclides are shown, and also F-18 to indicate reactor power. Cr-51 activity shown in blue greatly increased at the final phase of the shutdown, much more so than during the forced oxidation. Zn-69m clearly exhibits a different behavior than the other nuclides.

This $3^{\text {rd }}$ deployment also highlighted an interesting artifact at one of the 3 CZT detector locations. One of the outputs of the DA is total spectral count-rate. This output is shown Figure 10. It can be seen that there were multiple very significant but short term spikes in count-rate over a $\sim 3$ day period. But when all the nuclide results were examined, there was no increase in nuclide activity for any nuclide. But the MDA of some of the low-energy nuclides also showed increases during this 2-3 day period e.g. $\mathrm{Cr}-51$ in the bottom graph. Examining spectra before, during, and after the spikes showed no gamma peaks, but during the spikes did show an increase in low-energy counts. This is consistent with scattered radiation. The initial suspicion was that radiographic welding examination tests were happening, but this could not be confirmed by the plant. Later, it was determined that fuel transfers were being made at about that time, and that the
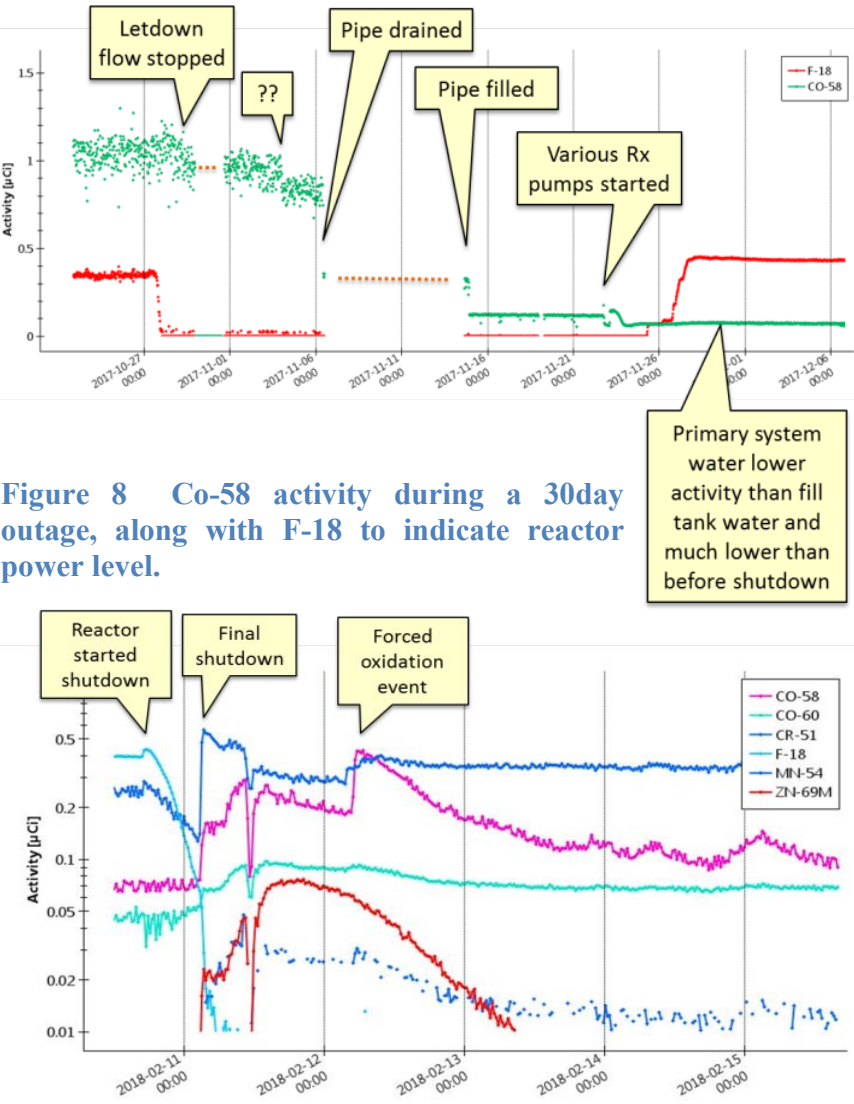

Figure 9 Activity of 5 key nuclides plus F-18 during the first 4 days after shutdown.

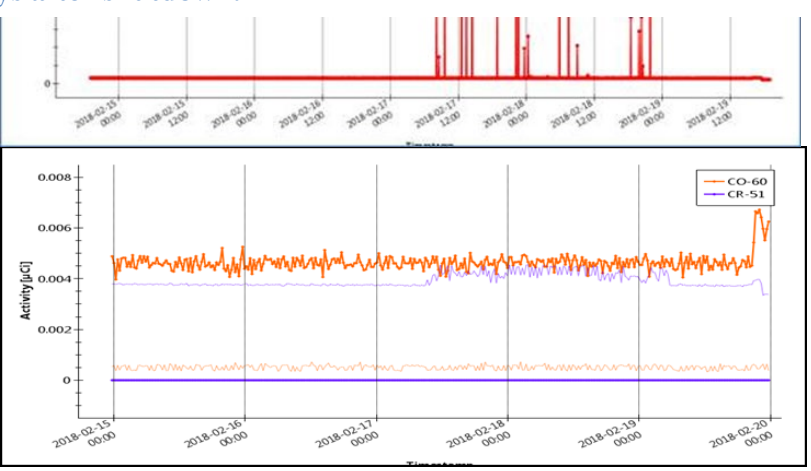

Figure 10 Top graph shows total count-rate in cps. Bottom graph shows the activity and MDA for Co-60 and Cr-51. The activity of $\mathrm{Cr}-51$ is not detectable, and the thin blue line at the middle of the graph is the MDA. The increase in MDA correlates in time to the period of spikes in count-rate. fuel transfer canal was very close to this particular measurement point. This highlights that many 
different events may highly impact the radiation fields in any given plant area. For a more detailed description of the reactor events causing these changes, see reference 12.

The data set in Figure 11 highlights just one example illustrating the advantage of executing multiple parallel workflows in the DA. Here each workflow has the same analysis parameters, only the acquisition times are different. Shorter acquisition times enable a quick review of the results - the data is available immediately after the measurement, and without the delay of sample collection, transfer to the lab, counting, and analysis. In addition, these short measurements allow the identification of transients that would be missed by 2 -hour and 12 -hour acquisitions here, and certainly by the

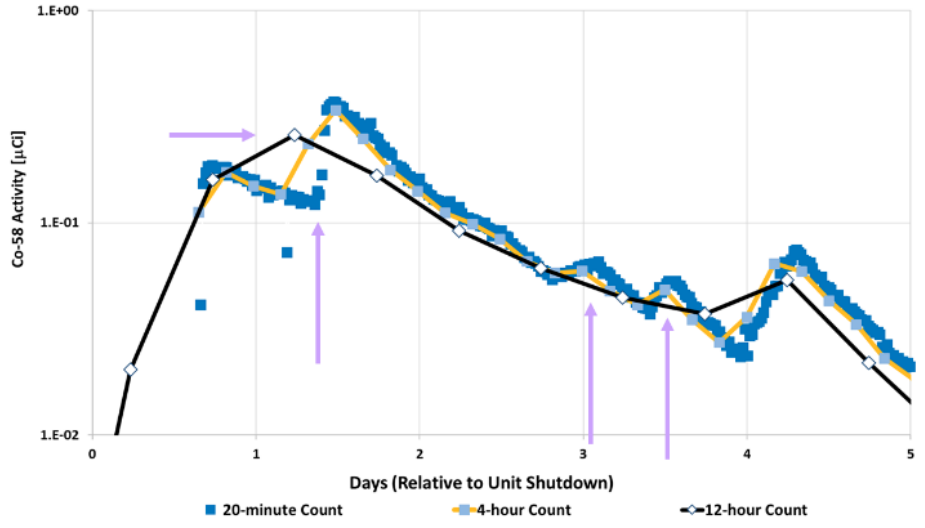

Figure 11 The same overlaid data but with 20 minute, 2 hour, and 12 hour acquisition times. The 20 minute data clearly shows the transients not seen in the $\mathbf{2}$ hour or 12 hour data. However the longer counts show more lower-activity nuclides and better precision. daily plant grab samples.

The last example of a reactor application of continuous sequential gamma spectroscopy is at a radionuclide production facility. Two stack gas effluent monitor using the DA have been built and installed and a third is under construction. The gas sample is extracted from the stack, filtered to remove particulates and iodine, and then passed continuously through a 17 liter Marinelli Beaker inside a lead shield. A $30 \%$ relative efficiency HPGe detector is used to assay the sample. Workflows analyze the data continuously every 10 minutes, 1 hour, and 1 day. Figure 12 shows the first system. Figure 13 shows 9 days of activity - over the Christmas Holidays. The wide dynamic nature of the stack gas concentration is very obvious. The total dynamic range of the measured data is nearly 7 decades, and neither the upper limit nor the lower limit have yet been reached.
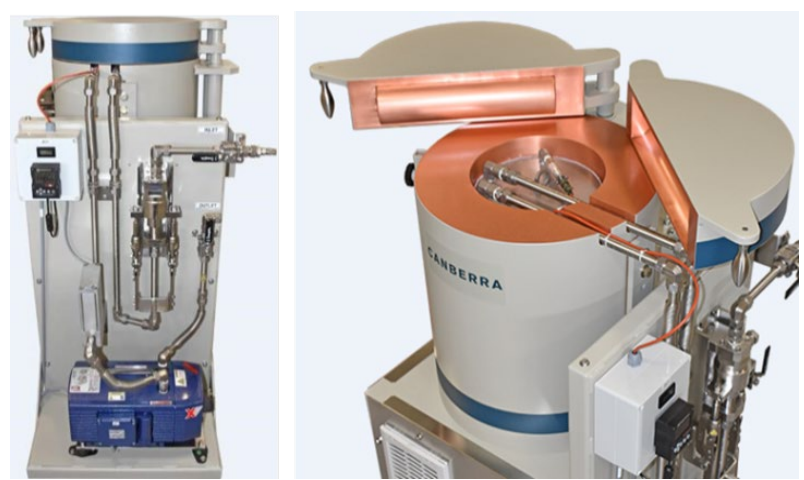

Figure 12 Stack Gas Monitor at research reactor used to produce medical radioisotopes. Sample container is visible in image of the opened shield at the right. An HPGe detector [not visible] is inserted in the middle of the sample chamber.

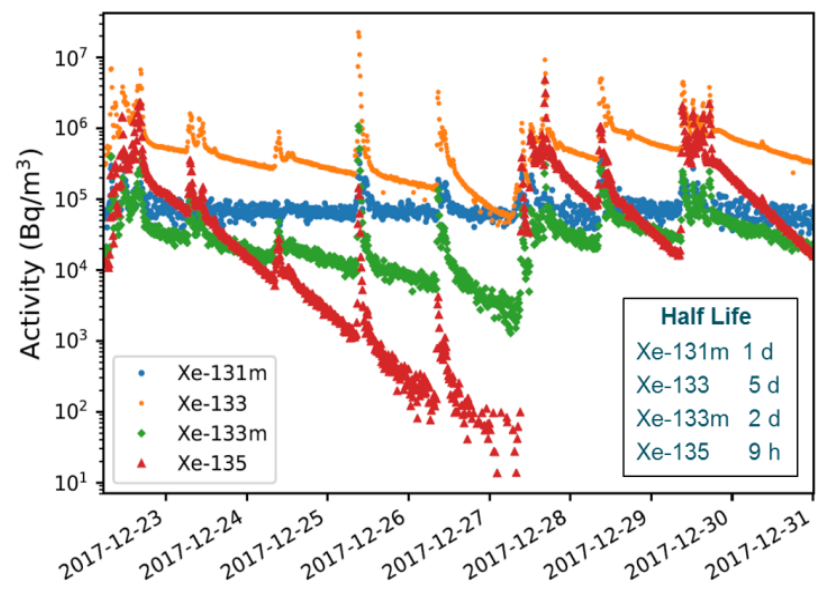

Figure 13 Activity of stack gas effluent at isotope production reactor facility.

\section{Beneficial Applications of Continuous Quantitative Gamma Spectroscopy at Operating Nuclear Power Plants and D\&D Projects}

Many opportunities may exist in NPP operations where installed [permanently or temporarily] gamma spectroscopy units may assist or help optimize plant operations. Many of these opportunities still need to be tested or demonstrated to document their benefits. 
- Real-time radiation field monitoring inside plants at areas causing worker dose (as highlighted in the present paper):

- Enhances outage and ALARA planning - optimizes critical path activities

- Prevents or minimizes entering into unforeseen radiological conditions

- Improves classification of radiological areas

- Forecasts when areas with higher radiation fields can be entered or down posted

- for instance, if a plant had a fuel leak, a steam leak in a confined space could create an unnecessarily high exposure to the worker in the early outage days but will be much lower with a short waiting period because of the iodine and noble gas isotope decay

- Primary coolant monitoring in pipes during operation

- Could be done on the liquid phase in PWRs. For BWRs, could be on the liquid phase or gas phase

- Provides nuclide-specific corrosion product trending

- Builds a database for trending and mining routines for retrospective evaluations or realtime optimizations

- Could allow improved core performance. Movement of corrosion products as detected by changes in their ratios can give early indications of crud-induced power shift or crudinduced localized corrosion activity transport.

- Could allow early detection of fuel cladding damage, e.g. by looking at radio-iodines, noble gases and their ratios.

- Better and faster fuel cladding defect localization during local flux depression operations. Instant feedback of results, rather than the delay and expense of frequent grab samples and subsequent laboratory analysis.

- Monitoring for control rod leakage by evaluation of the ratios of $\mathrm{Ag}, \mathrm{Sn}$, and In radionuclides.

- Moisture carry-over measurements to evaluate issues with the moisture separator or steam dryer in BWRs by monitoring for $\mathrm{Na}-24$ or $\mathrm{Co}-60$ in the steam exiting the core.

- Primary-secondary leaks and leak-rates

- Early detection by the increased presence of certain nuclides on secondary side

- Ratio of key nuclides from sample measurements on both sides gives leak-rate

- Primary coolant monitoring during early shutdown to see when workers can safely begin maintenance operations, thus minimizing critical path time

- A common criteria is that certain nuclides [e.g. Co-58 at PWRs or Co-60 at BWRs] should be below an EPRI recommended value or plant-specific limit; e.g. $0.5 \mathrm{uCi} / \mathrm{cc}$.

- Current practice is to take grab samples every 4-6 hours for subsequent laboratory analysis an hour or so later. On-line measurements could give these results immediately, and allow work to begin potentially 5-7 hours earlier, which is a significant critical path savings.

- $\quad$ Fuel leak detection

- In BWRs, continuous monitoring and reporting of Xenon nuclide activity and key nuclide ratios during local flux depression to identify region with the leaking fuel bundle.

- In PWRs, continuous on-line monitoring of lodine nuclide activity and "effective lodine"

- Monitoring of liquid effluents from the plant

- Continuous recording of nuclide-specific activities rather than gross count-rate.

- Nuclide-specific activities can be used to take some action - e.g. activate controls to stop release if above limit.

- Nuclide-specific activities can create alarm settings more closely correlated to dose than by gross gamma count-rate. 
- Continuous measurements prove that nothing happened between the times grab samples were taken.

- For very low MDAs, the many short continuous measurements can be integrated into a very long period.

- Monitoring of gaseous effluents from the plant

- Continuous recording of nuclide-specific activities rather than gross count-rate.

- Nuclide-specific activities can be used to take some action - e.g. activate controls to stop release if above limit.

- Nuclide-specific activities can create alarm settings more closely correlated to dose than by gross gamma count-rate.

- Continuous measurements prove that nothing happened between the times grab samples were taken.

- For very low MDAs, the many short continuous measurements can be integrated into a very long period.

- Spectroscopic continuous air particulate and lodine monitors

- Allows plant-specific nuclides to be accurately measured in the presence of Radon, and in the presence of the elevated and changing plant-caused background levels.

- Allows all gamma emitting nuclides to be measured, not just the ones within a few simplistic Region Of Interest boundaries on standard monitors.

- Mapping of nuclide-specific radioactivity on soil or surfaces

- The appropriate detector and DA can be integrated onto a moving platform. The DA has a GPS unit, or it can import location information from an outside source. The location is correlated with the assay results to allow for nuclide-specific area maps to be created.

- Can be used to provide data about spills to comply with requirements for database of accidents for future decommissioning,

- Can be used to map walls or floors or soil outside during Decontamination work.

- Activity of resins during sluicing operation

- Aim collimated detector at hose from filter tank to waste storage tank transporting the resin.

- Continuous measurements can be used to demonstrate nuclide homogeneity.

- Integral of all the continuous measurements is the total activity transferred to the storage tank

- Minimizes need to take resin samples back to lab

- Less dose and risk

- Total quantity measurement eliminates "non-representative sample" error

- Activity of effluent from fuel cleaning operations

- Aim collimated detector [probably under water] at effluent from fuel cleaning machine

- Continuous measurements show nuclide quantity and nuclide type variation with location on fuel element

- Measurements from the same area over time demonstrated that the cleaning operation can stop

- Nuclide-specific area dose-rates

- An unshielded nuclide-specific gamma detector [e.g. the small CZT detector-MCA] continuously generating spectral results can document over time which nuclides are causing the worker dose.

- The EcoGamma, a wide-range dose-rate meter, can plug into the DA and provide accurate dose-rate readings correlated with the nuclide results.

- Results can be correlated with plant operations to give quick indication of normal or abnormal operation.

- Multiple units distributed around the plant can reduce the need for HP techs to take readings. 
- Unit can transmit results over low-speed wireless tele-dosimetry network for easy adhoc installations in temporary work areas.

- Chemical decontamination unit or floor/wall decontamination unit

- Aim detector at effluent from device.

- Continuous measurements show nuclide quantity and type variation with location on fuel element or floor/wall.

- Stop the cleaning process or reduce the chemicals when measurements show output is low enough to meet requirements.

- Conveyor unit for soil, rubble, or other potentially contaminated items

- Aim collimated detector at items on conveyor.

- If nuclide activity is above pre-determined alarm level for each nuclide:

- stop conveyor and remove it; a low cost solution if this doesn't happen very often

- activate a diversion mechanism to separate the low from the high activity material; a fully automatic but more expensive solution

- Can use commercially available conveyor units

\section{Observations and Conclusions}

The internal walls of pipes containing radioactive fluids eventually become contaminated. If the objective is to measure the total radiation field from the pipe, as it was for this EPRI project, then the measurement methods used here were acceptable. But if the objective is to determine the activity in the coolant, and if there is significant pipe wall fixed contamination, then a different measurement geometry should be considered. One solution would be to extract a continuous sample of the fluid and measure it in a section of clean tubing that can be easily monitored for contamination, and either cleaned or easily replaced.

Primary coolant is a radiologically complex matrix. There are many nuclides present, and they cover a wide range of concentrations. Setting up the spectral analysis parameters and the nuclide libraries for CZT detectors requires very careful consideration. The energy resolution of the CZT detector used here was adequate for reliable detection of the major constituents, but certain nuclide combinations, and low levels of low energy nuclides were difficult and required careful setup by experts. The portability of the CZT detector allows it to be quickly setup and removed. But if one was considering an installed system for long term operations, a shielded and collimated HPGe detector is recommended.

The small shielded CZT detector is ideal for measurement of modestly radioactive materials or in modest dose rate areas. But the small size imposes limitations on low-level measurements. A normal HPGe detector can measure low activities with much shorter count times. And if the nuclide mixtures are not too complex, Nal detectors are available in very large sizes, perhaps for large area soil and floor mapping applications.

Furthermore, installed HPGe equipment using this technology demonstrated that wide dynamic range activities are measurable, and that the device can operate continuously and reliably over a 15 month period - as of this writing.

These projects have demonstrated the feasibility of collecting continuous measurement results of nuclide-specific gamma radiation fields, and activity of fluids such as primary coolant in pipes and effluent gas from stacks. But since these are not common applications at this point in nuclear facilities the realization of the benefits as envisioned to be gained by this data collection has yet to come to fruition. It is highly recommended that future demonstration projects be set up using HPGe detectors to viewing a continuous stream of extracted fresh primary coolant in a clean sample line. And that there be a companion project to evaluate the data from various nuclide ratios and correlation with other plant parameters to validate some of the expected advantages of this technology presented in section IV of this document. 


\section{REFERENCES}

1. "Remote Monitoring Technology Guide for Radiation Protection: Field Implementation of Remote Monitoring, 2015 Revision": Electric Power Research Institute [EPRI], Palo Alto, CA: 2015. EPRI document 3002005480.

2. "In-Plant Gamma Spectrometry: Isotopic Data Collection Experiences": EPRI, Palo Alto, CA: 2015. EPRI Document 3002005481.

3. "EPRI Pressurized Water Reactor Standard Radiation Monitoring Program 2014 Revision"; EPRI, Palo Alto, CA: 2014. EPRI Document 3002003155.

4. EPRI BWR Radiation Level Assessment and Control (BRAC) Program 2014 Revision": EPRI, Palo Alto, CA: 2014. EPRI Document 3002003157.

5. "Cobalt Reduction Sourcebook"; EPRI, Palo Alto, CA: 2010. EPRI Document 1021103.

6. "Radiation Field Control Manual"; EPRI, Palo Alto, CA: 2004. EPRI Document 1003390.

7. F. L. BRONSON, W. MULLER, J. ZICKEFOSE and C. HERMAN, "A Collimated CZT Detector for Quantitative Gamma Assays", Proceedings of Waste Management 2017, March 2017, Phoenix $A Z$.

8. J. BRIESMEISTER (ed.), "MCNP - A General Monte Carlo N-Particle Transport Code, Version 4a." Report LA-12625-M, Los Alamos National Laboratory (1993)

9. F. BRONSON, B. YOUNG, "Mathematical Calibrations of Ge Detectors and the Instruments that Use Them", Proceedings of 5th Annual NDA/NDE Waste Characterization Conference, Salt Lake City, UT, Jan 11, 1997.

10. F. BRONSON, "ISOCS, A Laboratory Quality Ge Gamma Spectroscopy System that you can Take to the Source for Immediate High Quality Results", Proceedings of the Rapid Radioactivity Measurements in Emergency and Routine Situations Conference, London, 15-17 October 1997

11. F. BRONSON, "Data Analyst - A Device for Continuous Repeating Real-Time Assay of HPGe, CZT, or Scintillation Gamma Spectra", Proceedings of Waste Management 2017, March 2017, Phoenix AZ.

12. C. GREGORICH, F. BRONSON, W. MELDRUM, B. SORBER, "Real-Time Gamma-Isotopic Radiation Field Characterization - In-Plant Demonstration"

Proceedings of the $21^{\text {st }}$ International Conference on Water Chemistry in Nuclear Reactor Systems, September 2018, San Francisco CA 